\title{
Isolation of a Corynebacterium and its Transitional Forms from a Case of Subacute Bacterial Endocarditis treated with Antibiotics
}

\author{
By RUTH G. WITTLER \\ Veterans Administration Central Laboratory for Clinical Pathology and Research \\ and Department of Bacteriology, Communicable Disease Division, Walter Reed \\ Army Institute of Research, Washington, D.C., U.S.A. \\ W. F. MALIZIA \\ Laboratory Service, Walter Reed General Hospital, Washington, D.C., U.S.A. \\ PATRICIA E. KRAMER, J. D. TUCKETT, H. N. PRITCHARD \\ AND H. J. BAKER \\ Department of Bacteriology, Communicable Disease Division, Walter Reed \\ Army Institute of Research, Washington, D.C., U.S.A.
}

(Received 7 April 1960)

\begin{abstract}
SUMMARY
A bacteriological study was made of blood specimens taken repeatedly during a 2-year period from a child with subacute bacterial endocarditis who received intensive treatment with antibiotics. The conventional bacillary form of Corynebacterium sp. was present in the blood and bone marrow of the patient before the beginning of antibiotic therapy and on occasions when the administration of antibiotics was suspended. These were the periods when the patient showed overt symptoms of clinical illness. When antibiotic therapy was adequate to produce clinical remission of symptoms, the infecting organism was not eradicated, but persisted in the blood in a small granule-like form that could be demonstrated and cultured only by highly specialized techniques. The cultural procedures required to bring about reversion of the granule-like form to the conventional bacillary form and the morphology of the various transitional forms that the organism assumed during the reversion process are described and illustrated.
\end{abstract}

\section{INTRODUCTION}

A recurrent problem in diagnostic bacteriology is the frequency of cultures which are negative for bacteria from the blood and body fluids of patients with febrile or inflammatory processes. A second and presumably related problem is the occasional appearance of slow growing, small, pleomorphic bacteria resembling corynebacteria in these otherwise negative cultures, particularly when the specimen originates from a patient with a chronic or recurrent illness. The isolation of diphtheroids or corynebacterium-like organisms from human blood, body fluids and tissues has a long history. The varied interpretations of their significance in disease can be traced back from the discussions and references given in recent papers by Kassel (1958), 
Carpenter, Howard \& Lehman (1956), Kassel \& Rottino (1955), Fleisher (1952), and Jiménez Díaz \& Arjona (1949), to the early work of Bunting \& Yates $(1913 a, b)$, Billings \& Rosenow (1913) and Cunningham (1917). A possible explanation for the apparently negative findings in some of these problem cases, and for the delayed appearance of the corynebacterium in others becomes available on the basis of the observations reported in this paper. We have followed for more than 2 years the case of a child with a congenital defect of the heart and intermittent fever who was diagnosed as having subacute bacterial endocarditis (SBE) on the basis of clinical findings and blood cultures positive for corynebacteria. Bacteriological studies demonstrated the continuous presence of various non-bacillary forms of the organism in the blood stream of the host during long periods of clinical remission and apparent recovery. The influence of antibiotic therapy on the morphology of the organism is described, and the relationship between the morphological form of the organism and the clinical course of the disease in the patient is discussed.

\section{METHODS}

Collection of specimens. Venous blood specimens were drawn from the arm in the usual way. The bone-marrow specimen was taken by sternal puncture. Before taking specimens meticulous care was observed in cleansing the skin at the site of the puncture with iodine and ethanol, and strictly aseptic procedures were employed during collection of the specimens.

Culture media. Each specimen was cultured on some of the following media as indicated in the text. (1) Bacto fluid thioglycollate medium (Thio) or BBL (Baltimore Biological Laboratory, Inc. Baltimore, Maryland, U.S.A.) fluid thioglycollate medium (Th-BBL) unenriched, or enriched with $20 \%(\mathrm{v} / \mathrm{v})$ normal rabbit serum. (2) BBL trypticase soy broth containing $0.1 \%(\mathrm{v} / \mathrm{v})$ agar (TSB). (3) BBL trypticase soy agar (TSA), enriched with $6 \%(\mathrm{v} / \mathrm{v})$ normal rabbit, horse, sheep or human blood. (4) Bacto veal infusion agar (VIA), enriched with $15 \%$ (v/v) normal horse or rabbit serum, with $15-20 \%(\mathrm{v} / \mathrm{v})$ normal human serum, or with $25 \%(\mathrm{v} / \mathrm{v})$ human ascitic fluid (Asc Fl). (5) Bacto veal infusion broth (VIB) enriched with $25 \%(\mathrm{v} / \mathrm{v})$ human ascitic fluid. (6) Bacto Casman medium base enriched with $6 \%(\mathrm{v} / \mathrm{v})$ normal rabbit blood. (7) A mixture of equal parts of Thio (or Th-BBL) and VIB enriched with horse or human serum or human ascitic fluid to the various final concentrations indicated in the text. (8) Fluid medium prepared from Bacto PPLO agar from which the agar was removed by filtration before heating (PPLOFl). The fluid medium was enriched with $25 \%$ (v/v) human ascitic fluid. (9) Embryonated chick eggs, between 7 and 11 days of age. (10) Chick chorioallantoic membrane (CAM) tissue cultures maintained in Hanks's balanced salt solution containing 10-15\% $(\mathrm{v} / \mathrm{v})$ normal rabbit serum.

Fermentation and biochemical tests were carried out by using the standard methods and media described in the Army Technical Manual (TM 8-227) of 1951.

The anaerobic sugar basal medium used for fermentation studies of microaerophilic cultures contained the following ingredients (g./l.): 10, trypticase; $2 \cdot 5$, sodium chloride; 0.5, sodium thioglycollate; 0.75 , Bacto agar; 0.02, phenol red; the medium was adjusted to $\mathrm{pH} \mathbf{7 \cdot 6}$.

Sera and ascitic fluid. The sera and ascitic fluid used for enrichment of media 
were Seitz filtered and stored in the frozen state. After thawing they were Seitz filtered a second time, heated at $60^{\circ}$ for $30 \mathrm{~min}$., and incubated before being added to the culture media.

Egg inoculation. Embryonated chick eggs, 7-11 days old, were inoculated with $0.1 \mathrm{ml}$. samples of the patient's blood, or with $0.1 \mathrm{ml}$. suspensions of pooled minced chorioallantoic membrane (CAM) or allantoic fluid from a previous serial egg passage of the blood specimen. The inoculum was dropped on the CAM or injected into the allantoic cavity. The eggs were sacrificed between the fourth and eighth day after inoculation. Harvests were made from the CAM and allantoic fluid. Preliminary tests had shown that amniotic fluid, amniotic membrane, and yolk-sac membrane were less suitable than CAM or allantoic fluid for passage of the organism under investigation. Harvested CAM tissues were minced before culturing on media or passing to fresh eggs. CAM and allantoic fluid harvests were tested for the presence of organisms by culturing on VIA plates containing $15 \%(\mathrm{v} / \mathrm{v})$ normal rabbit serum. The plates were examined for the development of deep granular colonies or macroscopic growth.

Tissue culture. CAM from 9- to 14-day-old embryonated chick eggs was harvested aseptically, finely minced with scissors, and suspended in Hanks's balanced salt solution containing $10 \%(\mathrm{v} / \mathrm{v})$ normal rabbit serum. Antibiotics were never added to the tissue cultures. The suspension of tissue was distributed in small glass tissueculture dishes (Warren, Wittler \& Vincent, 1955) containing a round glass coverslip in the bottom. Growth occurred from the minced explants and spread in monolayers on the coverslips. The CAM tissue cultures were inoculated on the fourth to seventh day of growth with $0.1 \mathrm{ml}$. of whole blood from the patient or with $0.2 \mathrm{ml}$. of the harvest from previously infected tissue cultures when serial passage was employed.

Staining methods. Essentially the same methods as those described by Wittler, Cary \& Lindberg (1956) were used. Microscopic observations were carried out on fresh specimens or cultures by using a Cooke, Troughton \& Simms phase contrast microscope with a long working distance condenser and oil-immersion objective. The material was either unstained or stained lightly with methylene blue and azur stain previously dried on the coverslip according to the method of Dienes (1939, 1942). Methanol-fixed preparations stained in Giemsa's solution buffered to $\mathrm{pH} 6 \cdot 8$ were also studied for comparison. Gram's method was used to detect conventional bacterial forms.

Tissue cultures grown on coverslips were stained in the dishes in which they were cultivated. After harvesting the supernatant fluids, the cultures were rinsed with two changes of Hanks's balanced salt solution and fixed with absolute methanol for $5 \mathrm{~min}$. after each rinse. Methanol was removed and replaced with Giemsa stain

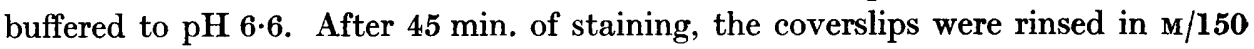
phosphate buffer at pH 6*6, dehydrated and mounted on slides.

Photography. Photomicrographs of living cultures by phase contrast were taken with a Bausch \& Lomb Model L camera and a Bausch \& Lomb tungsten ribbon filament illuminator. Kodak contrast process panchromatic sheet film was used with a transmission type interference filter (wavelength $550 \mathrm{~m} \mu$ ).

Controls. The inclusion of controls at each step of these experiments was deemed imperative to permit valid interpretation of the findings. For every plate, tube or bottle of culture medium inoculated with specimens or transfers of specimens, 
duplicate uninoculated plates, tubes or bottles of the same lot of medium were incubated along with the test cultures, opened for examination at the same time as the test cultures, and examined and transferred in exactly the same manner as the test cultures. On the rare occasions when a contaminant appeared in the control cultures, the test cultures for that particular series were discarded and the work repeated. Thus for every positive result reported in this study, it will be understood that duplicate uninoculated medium was incubated and examined by the same methods and found to be negative for any evidence of presence of organisms.

Furthermore, awareness of the possibility of encountering inclusions similar to those described by Nelson (1936), Pappenheimer, Molloy \& Rose (1945), or Van Herick \& Eaton (1945) in embryonated eggs led us to make a thorough search for such contaminating agents before using eggs or CAM tissue cultures for our experiments. By the use of the various cultural, microscopic and staining techniques described above, inclusion bodies could readily be demonstrated in tissue culture preparations of the CAM of eggs of certain flocks of chickens in the WashingtonMaryland area (Pl. 1, fig. 1). Moreover, passage of CAM tissues infected with these inclusions through a series of CAM tissue cultures enriched with rabbit serum resulted in reversion of the agent through a series of transitional stages to a coagulase negative Staphylococcus aureus. Eggs from infected flocks were not used in any experiments involving the patient's specimens.

When CAM tissue cultures were prepared, a large enough pool of membranes was used to allow for large numbers of control uninoculated cultures. These were stained at various times during the experimental period. The controls were also carried through numerous serial transfers to eliminate the possibility of a delayed appearance of inclusions during passage in the presence of serum or other nutrients. Separate controls containing individual substances, such as Hanks's balanced salt solution, serum or normal human blood, and various mixtures of these substances to duplicate those used for test specimens were always included with each experiment. For all experiments involving eggs or tissue cultures from eggs, the report here of positive results for test specimens implies, therefore, that the numerous controls for that specimen were consistently negative.

\section{RESULTS}

Blood or bone marrow specimens from a child with SBE were examined bacteriologically on twenty-three separate occasions over a period of more than 2 years during which time this patient was under close clinical observation. The results of the bacteriological studies and their correlation with the clinical findings are presented in the text and in Fig. 1. The infecting agent isolated from the patient was not consistently found in the bacillary form characteristic of its species and recognizable by conventional methods. Therefore the various non-bacillary forms, as well as the methods used for their demonstration or isolation, are each described separately in the text.

\section{Case history and clinical course}

The patient, S.S., a $4 \frac{1}{2}$-year-old white female, was first admitted to the hospital on 5 November 1957 with a chief complaint of intermittent fever of 4 weeks duration and diminished exercise tolerance of 7 months duration. At the age of 6 months a 
loud precordial murmur had first been noted, and a diagnosis of congenital heart disease was made. At the time of admission to the hospital the patient was acutely ill. Her temperature was $100 \cdot 6^{\circ} \mathrm{F}$., and a few petechiae on the chest and upper arms were noted. There was a harsh Grade IV apical systolic murmur. The spleen was slightly enlarged. The initial white count was 15,400 and the corrected sedimentation rate was 30. No antibiotics had as yet been administered. When positive blood and bone-marrow cultures were obtained, a diagnosis of interventricular septal defect with subacute bacterial endocarditis was made.

Administration of penicillin (3,600,000 units daily) and streptomycin (500 $\mathrm{mg}$. daily) was started on 7 November at which time her temperature had reached $103^{\circ} \mathrm{F}$. There was considerable improvement in the patient's condition by 12 November. Her spleen was no longer palpable, the petechiae were absent, her sedimentation rate was 13 and temperature $99 \cdot 8^{\circ} \mathrm{F}$. Streptomycin was discontinued, but penicillin was administered until 24 November. Four to five days after discontinuing penicillin, the patient relapsed, and her temperature rose to $103^{\circ}-104^{\circ} \mathrm{F}$. Blood cultures were positive for corynebacteria. Penicillin therapy was reinstituted and clinical improvement followed with a decrease in temperature to $99 \cdot 4^{\circ} \mathrm{F}$. by 3 December. The patient remained relatively symptomless except for a low grade fever. Oxytetracycline $(750 \mathrm{mg}$. daily) was administered in addition to penicillin from 10 December 1957 until 15 January 1958, and chloramphenicol (1500 mg. daily) along with penicillin was given from 15 January to 26 February. Daily penicillin was continued while the patient, who was then symptomless, was followed as an out-patient. She neglected to take penicillin for 3-4 days at the beginning of April 1958, and for a week thereafter ran a low grade fever and felt ill. The daily dosage of penicillin was decreased to 500,000 units from 25 August 1958 to the present. Although the patient has continued to appear symptomless, the bacteriological studies described below indicate that a micro-organism remains present in her blood, presumably in a latent state.

\section{Isolation and characterization of the original bacillary form of Corynebacterium $s p$.}

One bone marrow and three blood specimens were taken during the first 3 days of the patient's hospitalization. The specimens were cultured by routine bacteriological procedures in Thio and TSB media. Small white fluffy balls of growth (Pl. 1, fig. 2) appeared in both media after several days of incubation. Gram-stained smears of the balls showed small homogeneous Gram-positive rods resembling corynebacteria (Pl. 1, fig. 3).

Blood specimens (19 November and 3 December) cultured in Thio and TSB media, yielded identical small Gram-positive rods. These cultures, however, grew more slowly and required 5-8 days for the small fluffy balls of growth to become readily visible. The isolation results were confirmed by four different laboratories on quadruplicate specimens of blood drawn on 3 December. (These specimens were cultured in the respective laboratories of two of the authors, R.G.W. and W.F.M; in addition they were cultured by Lucille B. Robinson, Veterans Administration Hospital, Mt. Alto, Washington, D.C., and by Sylvia G. Cary and Martha C. Dail, Diagnostic Section of the Department of Bacteriology, WRAIR, Washington, D.C.) 
A variant morphological form developed when the original TSB cultures of the blood of 5 November were transferred to blood agar plates. The variant was Gramnegative, pleomorphic and filamentous with beads and swellings among the tangled loops (Pl. 1, fig. 4). Morphologically these organisms resembled Streptobacillus moniliformis, but serologically they were distinct. Agglutination tests were negative (a) using the Gram-negative variant and standard $S$. moniliformis serum and (b) using a stock strain of $S$. moniliformis and the patient's serum. The variant form was unstable, and after a series of transfers on culture media reverted to small Grampositive rods resembling corynebacteria.

The strains isolated from the bone marrow of 7 November and from the blood specimen of 19 November were tested for their pattern of biochemical reactions. Both were positive for fermentation of glucose, maltose, lactose, sucrose, salicin, and sorbitol. Both were negative for fermentation of mannitol, negative for production of indole and nitrite from nitrate, and negative for catalase. Litmus milk became acid in 3 days, then formed a clot. Colonies on sheep blood agar plates were slow-growing, small, raised, grey and non-haemolytic. The colonies were smooth at the time of primary isolation from the patient, but when the biochemical tests were conducted ( 2 weeks later) the colonies had become rough. The organisms were non-motile, non-spore-forming, small, Gram-positive rods, occurring in small clumps, and showing a tendency to palisade. They required blood or serum for optimal growth. The cultures were micro-aerophilic at the time of primary isolation, but grew aerobically after 2 weeks of transfer on culture media. The organism was identified as a Corynebacterium sp., but the species was not identical with any of the systematically defined species.

The fluorescent antibody technique was used as an additional test to differentiate the Corynebacterium sp. from Listeria. Labelled specific anti-Listeria serum did not fluoresce with the patient's organism.

Sensitivity tests were performed. The Corynebacterium sp. was sensitive to penicillin, streptomycin, oxytetracycline, chloramphenicol, novobiocin, ristocetin and gantrisin.

\section{Isolation and characterization of various transitional forms}

The blood cultures were examined for growth forms other than conventional bacilli for two reasons. As reported above, a Gram-negative filamentous variant had already been encountered during subculture of the original blood culture. In addition, there was the possibility that treatment with antibiotics might encourage the production of $L$-phase variants in vivo which might not be as sensitive as the conventional bacillus to the action of antibiotics. On seventeen occasions blood specimens examined for non-bacillary and other atypical morphological forms were positive. For convenience we shall term these non-bacillary forms 'transitional' forms and discuss the choice of this term later.

Deep granular colonies. Sixteen blood specimens (see Fig. 1) were positive for transitional forms designated as deep granular colonies. The patient's freshly drawn heparinized blood or plasma was spread on the surface of plates containing VIA enriched with $15-20 \%(v / v)$ normal human serum or $25 \%(v / v)$ human ascitic fluid and incubated in candle jars. Agar block preparations stained by the method of Dienes were examined daily with a phase contrast microscope. After 5-7 days 
of incubation many clusters of small granules were observed which appeared to grow down into the agar generally beneath the WBC layered on the surface. Many of the granules which made up these colonies appeared to be strung together and formed a continuous cone of growth that could be traced to a depth of $10-15 \mu$ below the surface (Pl. 2, figs. 5-7). The colonies increased in diameter during the second week of incubation, but they could not be propagated by transfer to solid or fluid media. A control blood specimen taken from one of the authors, sterile normal rabbit blood, and uninoculated control plates of the media did not produce this growth form.

The granular form appeared to remain viable when passed serially in embryonated chick eggs (7-11 days old); the patient's blood was inoculated into the allantoic sac. At each transfer the allantoic fluid and chorioallantoic membrane were cultured on plates of VIA and rabbit serum. Generally by the third serial egg passage the CAM yielded deep colonies of small granules on the plate cultures.

'Fried egg' type of colony. On three occasions (14 November 1957, 17 December 1957, and 21 January 1960) the blood cultures yielded colonies with dense granular centres growing down into the agar and lacy vacuolated peripheries composed primarily of small, medium or large round bodies. Some of the bodies were filled with granules, while others appeared as empty vacuoles. This growth form bore a striking resemblance to the so-called 'fried-egg' type of colony that is characteristic of L-phase growth. The colonies averaged $60-100 \mu$ in diameter, although many exceeded these limits. On the first two occasions the colonies developed on plates inoculated with 3-day-old broth cultures (VIB + Thio + serum + penicillinase) of the patient's blood. On the third occasion they grew on plates (VIA + AscFl) inoculated directly with the patient's plasma. Plate 2 , fig. 8 , shows the appearance of these colonies in an impression preparation stained with Giemsa solution. It was common to note that the central granular core which grew down into the agar adhered to the agar rather than to the slide when impression preparations were made. Thus stained preparations exhibited an empty hole in the centre of the colonies, whereas phase contrast examination of the agar block revealed the granular cores as well as the peripheral portions of the colonies.

The 'fried-egg' colonies were culturally delicate and unstable. They could not be maintained by serial transfer. The isolate of 17 December 1957 was slightly more stable than the other two and was successfully passed through a series of three transfers on agar before being lost. An effort was made to determine the antibiotic sensitivity of this growth form at the second transfer by using the impregnated paper disk method. No valid interpretation of the results could be obtained, however, because of the slow and scanty growth.

Intracellular inclusions. During the early course of the disease when Corynebacterium sp. was readily isolated from the blood by routine methods, it was common to find in the large mononucleated cells of the patient's blood, and sometimes in the polymorphonuclear neutrophils, many small round cytoplasmic inclusions. These inclusions were $0 \cdot 7-1 \cdot 4 \mu$ in diameter. Some cells had none or only a very few inclusions, but others had as many as 50-100 packed in the cytoplasm ( $\mathrm{Pl}$. 2, fig. 9). They were best observed by spreading freshly drawn blood or the buffy coat from plasma on agar plates. Agar block preparations stained with methylene blue + azur according to the method of Dienes were made immediately from the 
plates and examined by phase contrast. The inclusions appeared quite distinct from the normal types of granulation found in WBC. They were very difficult to distinguish in Giemsa-stained preparations. Their nature is not known. They may have been a type of non-specific 'toxic' granulation. Nevertheless, more thorough investigation of these forms is indicated for the following reasons. It was observed that the number of deep granular colonies which developed beneath WBC on agar plates was particularly high when the blood specimen contained many cells which showed cytoplasmic inclusions. Secondly, during previous investigations (Wittler, 1952; Wittler et al. 1956) many observations had been made on the appearance of bacilli, such as Haemophilus pertussis or Corynebacterium sp. in the cytoplasm of tissue cells. When the bacilli changed from rod-shaped to small round Gram-negative forms, their appearance and arrangement in the cytoplasm were so similar to the inclusions observed in the present studies, that the resemblance could not be overlooked. The possibility exists, therefore, that the cytoplasmic inclusions found in the patient's blood represented non-bacillary forms of the Corynebacterium sp.

A second type of cellular inclusion was revealed by tissue culture studies. The blood specimen collected on 11 April 1958 was frozen for 2 months until suitable tissue cultures were available. The cultures were prepared from CAM + Hanks's balanced salt solution + normal rabbit serum. The patient's blood was thawed, inoculated, and passed serially for four transfers in the tissue cultures. At the time of each harvest (on the sixth day following inoculation), the tissue cells were stained according to the method described earlier with Giemsa stain and examined for inclusions. The stained cells from the third and fourth passage showed a heavy infestation of small inclusions (Pl. 2, fig. 10). The inclusions were found in clusters around the edges of the cells and were estimated to be $c \cdot 0 \cdot 25-0 \cdot 75 \mu$ in diameter. Since the original inoculum was diluted by a factor of $1 / 20,000$ by the fourth serial passage, multiplication rather than mere survival of the organisms seemed probable. The absence of detectable cytoplasmic inclusions until after the sixth day of the third passage suggested, moreover, that considerable time for growth of the infectious agent was required before it reached a size visible in Giemsa-stained preparations.

Round bodies and pleomorphic forms from broth cultures. The various transitional forms that appeared in broth cultures were studied on ten occasions. During the first year of the study cultural methods for the propagation of the round forms in broth and microscopic methods for detailed morphological information were not sufficiently developed to yield complete results. For instance, the earliest blood specimens were cultured in VIB + Thio + horse serum. It was soon found that human serum gave better growth than horse serum, but the results varied, depending on the source of human serum. The final modification which gave optimal and consistent growth of the round bodies was a mixture containing VIB + Thio $+25 \%$ $(\mathrm{v} / \mathrm{v})$ human AscFl. However, growth was not maintained for long periods in this medium. Early specimens were cultured in VIB + rabbit serum, VIB + Asc Fl, and Thio. Although the Corynebacterium sp. grew well in these media, the round bodies did not. Thio $+20 \%(\mathrm{v} / \mathrm{v})$ rabbit serum was finally found to be the most suitable medium for maintaining the round bodies in a viable state and at times for promoting reversion of these forms to corynebacteria. Even in this medium the round forms could not be carried for more than about 2 weeks. 


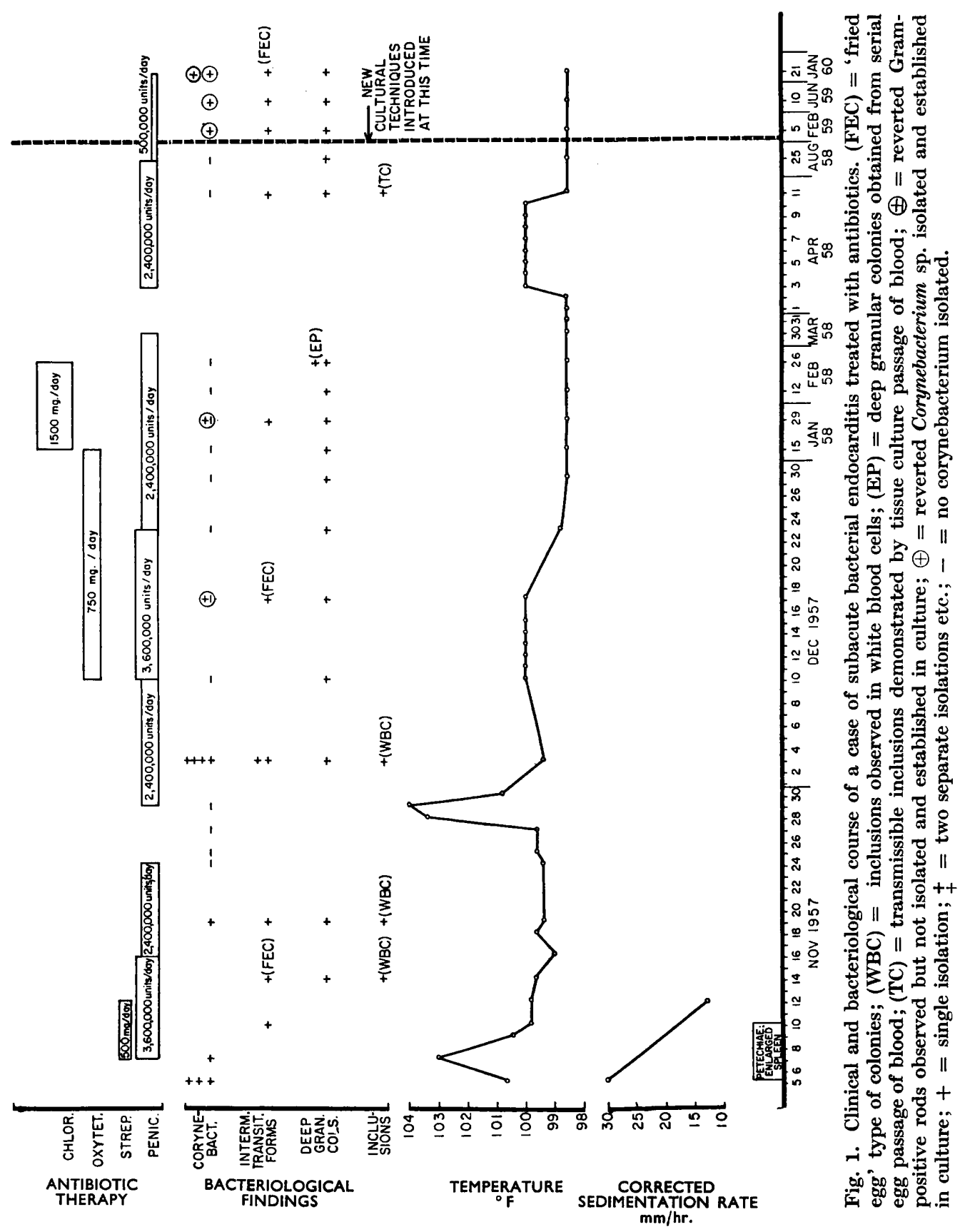


The transitional growth stage that consisted predominantly of round bodies produced a faint opalescent cloudiness in broth cultures within 3 days. The cloudiness was similar to that produced by Mycoplasma organisms (pleuropneumonialike organisms) in broth and unlike that produced by bacterial growth. At times it was so faint that it was scarcely detectable even when compared with uninoculated media. The growth was studied microscopically by placing a drop of the broth culture on a small agar block and examining it with a phase-contrast oil immersion lens. During the first few days of incubation, the organisms had a more or less spherical shape and were fairly uniform in size $(c$. 1.4-1.8 $\mu$ ). They were Gram-negative, but scarcely identifiable in Gram-stained preparations. Broth cultures stained with Giemsa solution at $\mathrm{pH} 6 \cdot 8$ showed deep rose coloured forms c. 1.2-1.6 $\mu$ in diameter. The shape of the bodies was readily distorted by the manipulative procedures involved in viewing them microscopically. Therefore it was concluded that possibly they lacked rigid cell walls.

It should be noted that on two occasions (Fig. 1; see 17 December 1957 and 29 January 1958) broth cultures that had shown only round bodies and no corynebacteria until the fifth day of incubation suddenly revealed a very few Grampositive rods resembling corynebacteria. The rods disappeared by the end of the following day and never reappeared, nor did any contamination ever develop in these tubes. In the light of later studies described below it is now believed that this puzzling observation might signify that a few of the round bodies had reverted spontaneously to corynebacteria, but that the reverted bacilli were too unstable to survive in those particular cultures.

In the second year of this investigation cultural techniques were finally devised that allowed further development of the round bodies and promoted their reversion to bacillary form. An adaptation of the methods of Gilmore \& Burnett (1959) was applied to the problem. Freshly drawn blood specimens (see Fig. 1, from February 1959 to January 1960 ) were inoculated into tubes containing VIB + Th(BBL) $+25 \%$ $(\mathrm{v} / \mathrm{v}) \mathrm{AscFl}+$ penicillinase. Within $48 \mathrm{hr}$. the tubes showed clusters of small round bodies. The mechanism of multiplication of this form is not yet known. Possibly there can be simple binary division of the bodies. This was suggested by the frequent observation of two bodies attached by a thin protoplasmic connexion (Pl. 3, fig. 11). When many of the bodies showed a phase-dense sector adjacent to a phase-transparent sector ( $\mathrm{Pl}$. 3, fig. 12) and when large numbers of these clusters were present (48-96 hr.), the tubes were transferred in toto to flasks containing $50 \mathrm{ml}$. PPLOFl+ $17 \mathrm{ml}$. AscFl + penicillinase. The small round bodies multiplied for 3-4 days after transfer, then seemed to become stationary, then appeared to decrease in number. A second transfer in PPLO Fl+AscFl with or without penicillinase was made generally after 3-5 days of incubation. On one occasion, however, this transfer was delayed until the twentieth day of incubation and still gave results positive for reversion. The morphology of the round bodies, followed in various media and by phase contrast microscopy or by Giemsa-stained preparations, underwent a series of changes. The bodies became irregular in shape and developed small protrusions (Pl. 3, fig. 13). Extremely fine short thread-like forms extended from the bodies or were found free in the medium (Pl. 3, figs. 14, 15). Sometimes individual bodies appeared to be divided up into packets of delicate, pale pink (Giemsastained) round or oval granule-like forms. At other times these packets were found 
lying next to a group of pleomorphic bodies (Pl. 3, figs. 16, 17). The clusters of larger round bodies merged into masses consisting of granules, vacuoles and seemingly structureless amorphous material (Pl. 3, figs. 18, 19; Pl. 4, figs. 20, 21). Reorganization of structure then followed and chains of granules and delicate beaded filaments appeared in the masses (Pl. 4, figs. 22, 23). The plump, coccoid, or pleomorphic bacilli which first emerged from the masses were Gram-negative or Gram-variable (Pl. 4, figs. 24, 25). With further incubation they became Gram-positive. Transfer to VIB + rabbit serum helped to stabilize the reverted culture, and the bacilli then assumed the smaller slender rod-shape typical of this Corynebacterium species.

It has not yet been possible to follow and photograph one particular organism through its whole developmental cycle from granule to bacterium. The fact that the stages illustrated here were observed repeatedly and that reversion to Corynebacterium sp. was consistently obtained from them is strong evidence that the various transitional forms were non-bacillary variants of the conventional bacillus.

The results obtained by using the patient's blood were compared with the results obtained by using a normal control blood. At the same time that the patient's blood was being cultured, blood from one of the authors (whose skin flora included corynebacteria) was drawn and cultured in the same batches of media and by identical transfer methods. A variety of artifacts appeared during incubation, but round bodies and other transitional stages morphologically similar to those in the test cultures never developed in the control series, and no corynebacteria were isolated.

To establish the actual source of the isolated corynebacterium another type of control experiment was performed. Swabs of the skin of the patient's arm were cultured. Several species of diphtheroids were isolated. These organisms were larger bacilli which grew luxuriantly within $24 \mathrm{hr}$. in Thio and TSB and formed large white colonies on blood agar plates. No transitional stages were found in any of the cultures of the skin diphtheroids.

\section{Characterization of the reverted Corynebacterium strains}

The four Corynebacterium strains which had reverted from transitional forms were compared. These were designated no. 52, obtained from the blood specimen of 5 February 1959; no. 6-4a from the blood specimen of 10 June 1959; no. M-5 from the specimen of 21 January 1960, isolated in the laboratory of one of the authors (W.F.M.); no. 11-4d from a duplicate specimen of 21 January 1960 isolated in the laboratory of another of the authors (R.G.W.). The results of biochemical and cultural examination of these strains are recorded in Table 1. The results on the original bone marrow and blood isolates of 1957 are included for comparison. The biochemical tests carried out in 1960 were started on the same day and were run in duplicate for each strain. Aerobic strains were tested for fermentative ability using broth and semi-solid serum-enriched trypticase basal medium. Micro-aerophilic strains were tested in serum-enriched trypticase + thioglycollate basal medium and in serum-enriched trypticase semi-solid basal medium.

Recognition of the remarkable variability of these Corynebacterium strains under laboratory conditions is essential to any interpretation of the biochemical and cultural results. For instance, no. 52 at the time of reversion to bacillary form was extremely rough. It was transferred twice weekly for 2 months in $20 \%(\mathrm{v} / \mathrm{v})$ rabbit serum broth and agar. The growth in broth became smooth, but dissociation 
into two types of colony variant occurred on agar. Plating directly from the broth cultures revealed only one colony type; it was similar to one of the agar variants but not identical. The smooth broth variant was stored in the refrigerator and in the deep freeze. After 1 month in the refrigerator the strain became extremely rough and could not be maintained in transfer. After 1 year in the deep freeze the strain became slightly rough. It is this slightly rough variant (52-3) that is characterized in Table 1.

Table 1. Biochemical reactions and growth characteristics of original and reverted Corynebacterium strains from blood or bone marrow

\begin{tabular}{|c|c|c|c|c|c|}
\hline $\begin{array}{l}\text { Strain } \ldots \\
\text { Date of } \\
\text { specimen ... } \\
\text { Date of test ... }\end{array}$ & $\begin{array}{l}\text { 7, } 19 \text { Nov. } 1957 \\
3 \text { Dec. } 1957\end{array}$ & $\begin{array}{r}5 \text { Feb. } 1959 \\
16 \text { Feb. } 1960\end{array}$ & $\begin{array}{l}10 \text { June } 1959 \\
16 \text { Feb. } 1960\end{array}$ & $\begin{array}{l}21 \text { Jan. } 1960 \\
16 \text { Feb. } 1960\end{array}$ & $\begin{array}{c}\text { Reverted } \\
\text { 11-4d } \\
\text { 21 Jan. } 1960 \\
16 \text { Feb. } 1960\end{array}$ \\
\hline Glucose & + & + & + & + & + \\
\hline Maltose & + & - & - & + & + \\
\hline Lactose & + & - & - & \pm & \pm \\
\hline Sucrose & + & - & - & + & \pm \\
\hline Mannitol & - & - & - & + & \pm \\
\hline Salicin & + & - & - & \pm & \pm \\
\hline Mannose & & + & \pm & + & $\bar{t}$ \\
\hline Rhamnose & & - & - & \pm & \pm \\
\hline Dextrin & & - & - & + & + \\
\hline $\begin{array}{l}\text { Galactose } \\
\text { Sorbitol }\end{array}$ & + & \pm & \pm & + & + \\
\hline Gelatin & & - & + & & \\
\hline Litmus milk & Acid, clot & Reduced & Reduced & - & - \\
\hline Urea & & - & - & - & - \\
\hline $\mathrm{NO}_{3}^{\prime} \rightarrow \mathrm{NO}_{2}^{\prime}$ & - & - & - & + & + \\
\hline Methyl red & & - & - & - & - \\
\hline Indole & - & $\overline{+}$ & + & + & + \\
\hline Catalase & - & + & + & + & + \\
\hline Motility & - & - & ${ }^{-}$ & - & Smonth \\
\hline $\begin{array}{l}\text { Serum broth } \\
\text { Horse, human } \\
\text { or sheep blood } \\
\text { agar }\end{array}$ & $\begin{array}{l}\text { Very rough } \\
\text { Small raised } \\
\text { grey rough } \\
\text { colonies } \\
\text { originally } \\
\text { smooth }\end{array}$ & $\begin{array}{l}\text { Sl. rough } \\
\text { Small raised } \\
\text { grey-white } \\
\text { colonies }\end{array}$ & $\begin{array}{l}\text { Rough } \\
\text { Small raised } \\
\text { grey-white } \\
\text { colonies }\end{array}$ & $\begin{array}{l}\text { Smooth } \\
\text { Small raised } \\
\text { grey smooth } \\
\text { colonies }\end{array}$ & $\begin{array}{l}\text { Smooth } \\
\text { Small raised } \\
\text { grey smooth } \\
\text { colonies }\end{array}$ \\
\hline $\begin{array}{l}\text { Haemolysis } \\
\text { Horse } \\
\text { Human }\end{array}$ & & $\begin{array}{l}+\beta \\
+\beta\end{array}$ & $\begin{array}{l}+\beta \\
+\beta\end{array}$ & $\begin{array}{l}+\beta \\
+\beta\end{array}$ & $\begin{array}{l}+\beta \\
+\beta\end{array}$ \\
\hline Sheep & - & - & - & - & - \\
\hline Rabbit & - & - & - & - & - \\
\hline $\mathrm{O}_{2}$ requirement & $\begin{array}{l}\text { Aerobic, } \\
\text { originally } \\
\text { micro-aero- } \\
\text { philic }\end{array}$ & Aerobic & Aerobic & $\begin{array}{l}\text { Micro-aero- } \\
\text { philic }\end{array}$ & $\begin{array}{l}\text { Micro-aero- } \\
\text { philic }\end{array}$ \\
\hline
\end{tabular}

Strain 6-4a was also extremely rough at the time of reversion to bacillary form. Passage on agar again resulted in dissociation into two types of colony variants. Truly smooth variants from this strain have never been obtained. One of the rough variants (6-4a-3) from broth cultures is characterized in Table 1. In addition to the changes in cultural characteristics, marked differences in the biochemical 
characteristics of the variants occurred. Comparison of the three variants from each of the two strains are recorded in Table 2. It will be noted that the variants (52-2 and 6-4a-2) which grew as tiny colonies and required micro-aerophilic conditions reduced nitrate to nitrite; the other variants lacked this property. Furthermore, the variant $(6-4, a-2)$ which became very rough lost the ability to produce catalase. Variant 6-4a-3 produced indole when the test was performed on cultures that were first carried through 10-12 rapid transfers in serum + thioglycollate medium.

Table 2. Biochemical reactions and growth characteristics of variants of two Corynebacterium strains isolated from blood

\begin{tabular}{|c|c|c|c|c|c|c|}
\hline \multirow{2}{*}{$\begin{array}{ll}\text { Strain } & \ldots \\
\text { Variant } & \ldots\end{array}$} & \multicolumn{3}{|c|}{52} & \multicolumn{3}{|c|}{$6-4 a$} \\
\hline & 1 & 2 & 3 & 1 & 2 & 3 \\
\hline Date of test ... & May 1959 & May 1959 & Feb. 1960 & May 1959 & May 1959 & Feb. 1960 \\
\hline Glucose & + & + & + & - & + & + \\
\hline Maltose & + & + & - & - & + & - \\
\hline Lactose & - & - & - & - & - & - \\
\hline Sucrose & - & - & - & - & - & - \\
\hline Mannitol & - & - & - & - & - & - \\
\hline Salicin & - & - & - & - & - & - \\
\hline Sorbitol & - & - & & - & - & \\
\hline Gelatin & - & - & - & & & + \\
\hline Litmus milk & Sl. alk. & Sl. alk. & Reduced & - & Proteolysed & Reduced \\
\hline Urea & - & - & - & - & - & - \\
\hline $\mathrm{NO}_{3}^{\prime} \rightarrow \mathrm{NO}_{2}^{\prime}$ & - & + & - & - & + & - \\
\hline Indole & - & - & - & - & - & + \\
\hline Catalase & + & + & + & + & - & + \\
\hline Motility & - & - & - & - & - & - \\
\hline Tellurite & $\begin{array}{l}\text { Shiny black } \\
\text { colonies }\end{array}$ & No growth & & No growth & No growth & \\
\hline Serum broth & Smooth & Smooth & Sl. rough & Sl. rough & Very rough & $\begin{array}{l}\text { Rough to sl. } \\
\text { rough }\end{array}$ \\
\hline $\begin{array}{l}\text { Sheep blood } \\
\text { agar }\end{array}$ & $\begin{array}{l}\text { Small raised } \\
\text { grey-white } \\
\text { colonies }\end{array}$ & $\begin{array}{l}\text { Tiny } \\
\text { translucent } \\
\text { colonies }\end{array}$ & $\begin{array}{l}\text { Small raised } \\
\text { grey-white } \\
\text { colonies }\end{array}$ & $\begin{array}{l}\text { Small raised } \\
\text { grey-white } \\
\text { colonies }\end{array}$ & $\begin{array}{l}\text { Tiny } \\
\text { opaque } \\
\text { colonies }\end{array}$ & $\begin{array}{l}\text { Small raised } \\
\text { grey-white } \\
\text { colonies }\end{array}$ \\
\hline $\begin{array}{l}\text { Haemolysis } \\
\text { (sheep blood) }\end{array}$ & - & - & - & - & - & - \\
\hline $\begin{array}{l}\mathrm{O}_{2} \text { require- } \\
\text { ment }\end{array}$ & Aerobic & $\begin{array}{l}\text { Micro-aero- } \\
\text { philic }\end{array}$ & Aerobic & Aerobic & $\begin{array}{l}\text { Micro-aero- } \\
\text { philic }\end{array}$ & Aerobic \\
\hline
\end{tabular}

In contrast to these examples of variation are the consistent results obtained on strains M-5 and 11-4d of January 1960 (Table 1). Great precautions were taken to avoid the production of variations by laboratory manipulation. The same methods for preparation of media, inoculation, transfer, and examination of cultures were followed with the duplicate specimens of January 1960, but all the procedures were carried out quite independently in two separate laboratories. The reverted Corynebacterium spp. were maintained by rapid transfer in serum-enriched thioglycollate medium and tested for biochemical characteristics within a week after reversion occurred. As shown in Table 1, these two strains were identical. Both M-5 and 11-4d were smooth and micro-aerophilic as were the original blood and bone marrow isolates of 1957 before they were carried in transfer. The pattern of sugar fermentations for the 1957 and 1960 isolates shows a discrepancy only in 
regard to mannitol. However, the results for litmus milk, nitrite from nitrate, indole and catalase are different for the 1957 and 1960 isolates.

In view of the results on the variants of strains no. 52 and no. 6-4a, which behaved quite differently with respect to litmus milk, nitrite from nitrate, indole and catalase, one cannot conclude that the 1957 and 1960 isolates were necessarily different species of corynebacteria. For instance, M-5 and 11-4d which were microaerophilic produced nitrite from nitrate as did the micro-aerophilic tiny variants 52-2 and 6-4a-2. M-5 and 11-4d produced indole as did 6-4a-3 after it was passed in serum + thioglycollate medium. The 1957 blood and bone marrow strains, tested after they became very rough, did not produce catalase, the same being true for the very rough 6-4,a-2 strain. For the difference in the litmus milk reaction of the 1960 strains there is no comparable parallel among the 1959 variants.

None of the strains isolated from the blood of the patient conformed entirely to the characteristics of the species already identified systematically. Our strains are probably more closely related to Corynebacterium acnes than to other taxonomically defined species of Corynebacterium. Until further studies can be completed, it is probably best not to use a species name.

Penicillin sensitivity tests were carried out on strains M-5, 11-4d, 52-3, and 6-4a-3. M-5 and 11-4d were sensitive to $0 \cdot 1$ units penicillin $/ \mathrm{ml}$., whereas the two rougher variants, $52-3$ and $6-4 \mathrm{a}-3$, were sensitive to $0 \cdot 4$ units $/ \mathrm{ml}$. and 1.58 units $/ \mathrm{ml}$., respectively. The very rough variant $6-4 \mathrm{a}-2$ was resistant to 25 units penicillin/ ml. Strain 52-3 was tested against 18 other antibacterial agents and was resistant only to gantrisin.

\section{DISCUSSION}

The foregoing results point to a correlation between the morphological form of the infecting microbe and its pathogenic potential in the infected host. The evidence for this correlation rests upon the following observations. When the patient was admitted to the hospital before receiving any antibiotic therapy, she was febrile and clinically ill. At this time the corynebacterium isolated from her blood and bone marrow was in its conventional bacillary growth form and could be cultivated on routine blood culture media. When antibiotics were administered, the patient's temperature declined and her clinical condition improved. Blood cultures then yielded a small granular form and other transitional stages of the corynebacterium which bore no resemblance morphologically or culturally to the conventional bacillary form. Nevertheless, these transitional stages were capable of reversion to the bacillary form of the corynebacterium when cultivated on appropriate media. When antibiotic therapy was interrupted, the patient suffered a recurrence of fever and illness, and the bacillary form of the organism reappeared in her blood. As soon as antibiotic therapy was resumed, the patient became symptomless, the bacillary form disappeared from her blood, but the granular form persisted. During a period of nearly 2 years of daily penicillin treatment the patient had no recurrence of symptoms, although the transitional stage of the organism remained present in her blood and retained its capacity to revert in special culture media. Furthermore, the reverted corynebacterium remained a 'penicillin-sensitive' organism when tested by the conventional antibiotic sensitivity techniques. From these observations the transitional stage of the corynebacterium appeared to be the form associated with 
the latent stages of the infection, whereas the bacillary form appeared to be associated with the active stages of the infection.

The possibility of the survival of bacteria in vivo in a latent or unrecognizable form is not a new concept, but experimental evidence to support this theory has been fragmentary. Our findings offer further experimental support for a hypothesis presented by McDermott (1958) to account for certain phenomena of 'microbial persistence' during antibiotic therapy, i.e. 'that there was something different about parasites when they were actually producing disease which made them more drug-susceptible and that micro-organisms which were not drug-resistant in the usual sense were nonetheless capable of surviving drug therapy in the tissues of man, so that antimicrobial therapy was seldom predictably eradicative'. And further, 'that there is a state of drug-indifference, that it is reversible depending on environmental changes, and that such states can be induced or favoured by the nature of the environment'.

Implications of the importance of the small granular filterable phases of bacteria to latency, recurrences and the carrier state of disease are found in KlienebergerNobel's (1951) discussion of these forms. However, in her review clear-cut definitions distinguishing between the true L-phase and the granular or filterable stages of bacteria were not given. Since that time, the concept of the distinction between the two forms has been clarified. According to her latest suggestion (Dr E. KlienebergerNobel, personal communication), the $\mathrm{L}$-phase is a stable growth form which no longer reverts to its original bacterial form. L-phase growth is described as a laboratory phenomenon found in vitro but not in vivo which, by appropriate cultural methods, reveals highly characteristic colonial and morphological features. On the basis of this definition the various growth phases of the organism described in the present study could not be termed L-phases of corynebacterium, since they were all highly unstable on culture media and since some of the stages were found in vivo. Moreover, they were morphologically diverse and included minute granular forms, small round bodies, and a wide range of non-bacillary pleomorphic forms. Nevertheless, some of the stages on culture media were temporarily capable of producing unstable colonies with deep granular centres and lacy vacuolated peripheries which resembled the L-phase of growth in their colonial and morphological characteristics. Furthermore, individual transitional stages were morphologically similar to certain stages of the L-phase of growth as illustrated and described by Klieneberger-Nobel (1949). Other stages appeared similar to the protoplasts illustrated and described by Fitz-James (1958; see his figs. 8-11) and by Spiegelman et al. (1958; see their figs. 3-5 and 9). All the varied stages of the organisms found both in vitro and in vivo in the present work we term 'transitional forms' to distinguish them from both the conventional bacillary form of the organism and from the stable laboratoryproduced L-phase of growth. It is our opinion that the in vivo transitional forms reported here and shown, at least in this case, to be of significance in the course of the disease, might well be analogous to the 'filterable' or granular forms of bacteria which Klieneberger-Nobel (1951) postulated might play a role in certain recurrent or latent infections.

The observations made on the patient S.S. are not unique. Since the investigation of the first case, we have had the opportunity to study similar cases of suspected endocarditis in three male patients (one child and two adults) all of whom had 
received previous antibiotic treatment. Blood and bone-marrow cultures were negative by routine cultural methods in each case. By the use of our special methods, however, transitional forms of a corynebacterium were cultured from the blood and bone marrow of these three patients. Reversion of the transitional forms to the conventional bacillary form was obtained by further culture on appropriate media. It was impossible to follow the latter patients for long periods and to carry out complete cultural studies on a series of specimens taken repeatedly from each patient. Nevertheless, all of the results obtained in the subsequent cases were in accord with the findings reported for the original case.

The accumulated evidence does not support the hypothetical contention that the Corynebacterium sp. might have been a contaminant from the laboratory or from the skin of the patient. The organism could not have been a laboratory contaminant, because duplicate specimens cultured in different laboratories yielded the same organism. Moreover, the enormous number of controls studied during the course of this investigation showed a very low rate of contamination and never yielded transitional forms. All cultures of the patient's blood yielded transitional or bacillary forms of corynebacterium. If this organism were a laboratory contaminant, one would expect all or most of the controls likewise to be positive for such transitional or bacillary forms. There is also good evidence that the source of the organism was not the skin of the patient. If the act of piercing the skin with a hypodermic needle were sufficient to introduce a corynebacterium into the blood sample, it would be expected that the blood sample from the author who served as a control subject would likewise have yielded corynebacteria. This did not occur. Furthermore, the skin organisms from the patient were not found as transitional forms in culture, whereas the organisms cultured from the blood specimens were usually found as transitional forms. The latter would appear, therefore, to be from a source other than the skin. Finally, the patient showed acute symptoms of illness of a clinical degree when the organism was isolated in its bacillary form. She appeared symptomless when the organism was present only as transitional forms. This correlation indicates that the source of the bacilli was systemic not superficial, that they must have been isolated from the blood and not from the skin.

The negative results for isolation of corynebacteria obtained by Kassel \& Rottino (1955) when they used germ-free techniques could, we believe, be explained on the basis of the cultivation methods they used. In our hands transitional stages incubated in the presence of crystal violet in PPLO broth for the first 5 days were unsuitable for further culture and reversion to corynebacteria. Therefore, if transitional forms were present in Kassel \& Rottino's specimens, their methods would not have enabled them to demonstrate and promote the propagation and reversion of these forms.

The results of the study on the biochemical characteristics of the Corynebacterium strains showed that the strains were capable of marked variation. The results did not necessarily prove that the strains did not belong to the same species. Our observations accumulated during these years of study on the strains from the blood lead us to believe they are all members of the same species. We showed that variations could readily be produced by laboratory manipulation. The variations in morphology suggest that other variations may occur also in vivo when the host environment changes during antibiotic treatment. The demonstration of the varia- 
tions among the isolates indicates, therefore, an area for further study both taxonomically and serologically, before valid criteria for establishing identity or lack of identity of Corynebacterium spp. are available. Quite aside from any value these studies may have in assisting in the identification of the etiological agent in any specific case, we believe their primary contribution lies in the means offered for demonstration in the host of viable agents of potential pathogenicity which until now might easily escape detection, or might be dismissed as contamination of a particular culture if the appearance of growth were long delayed.

The authors wish to thank Mrs Lucille B. Robinson (Veterans Administration Hospital, Mt. Alto, Washington, D.C.) and Miss Sylvia G. Cary and Miss Martha C. Dail (Department of Bacteriology, Walter Reed Army Institute of Research), for their confirmatory bacteriological and serological studies; the staff of the Paediatric Service of the Walter Reed General Hospital, especially Colonel L. J. Geppert and Major R. J. Hall, for the clinical data on the patient and for their interest and collaboration during this investigation; Miss E. Louise Craig (Medical AudioVisual Department, Walter Reed Army Institute of Research), for her generous assistance and advice on the photography; and the Medical Audio-Visual Department, Walter Reed Army Institute of Research and the Medical Illustration Section of the Walter Reed General Hospital for a number of the photographs.

\section{REFERENCES}

Bilingas, F. \& Rosenow, E. C. (1913). The etiology and vaccine treatment of Hodgkin's disease. J. Amer. med. Ass. 61, 2122.

Bunting, C. H. \& Yates, J. L. (1913a). Cultural results in Hodgkin's disease. Arch. int. Med. 12, 236.

Bunting, C. H. \& Yates, J. L. (1913b). An etiologic study of Hodgkin's disease. J. Amer. med. Ass. 61, 1803.

Carpenter, C. M., Howard, D. H. \& Lehman, E. L. (1956). Preliminary characterization of a group of unidentified pleomorphic bacteria isolated from blood and other tissues. J. Lab. clin. Med. 47, 194.

Cunningram, W. F. (1917). The status of diphtheroids with special reference to Hodgkin's disease. Amer. J. med. Sci. 153, 406.

Dienes, L. (1939). 'L' organisms of Klieneberger and Streptobacillus moniliformis. J. infect. Dis. 65, 24.

Drenes, L. (1942). The significance of the large bodies and the development of $L$ type of colonies in bacterial cultures. J. Bact. 44, 37.

Fitz-JAmes, P. C. (1958). Studies on the morphology and nucleic acid content of protoplasts of Bacillus megaterium. J. Bact. 75, 369.

Fleisher, M. S. (1952). Significance of diphtheroid micro-organisms in blood cultures from human beings. Amer. J. med. Sci. 224, 548.

Gilmore, E. \& Burnett, G. W. (1959). Isolation and maintenance of oral L forms of bacteria in liquid media. J. Bact. 77, 147.

Jiménez Díaz, C. \& Aruona, E. (1949). A new germ not described before, in subacute abacterial endocarditis (Corynebacterium endocarditis). Bull. Inst. med. Res., Madrid, $2,71$.

KASSEl, R. (1958). Etiological considerations in Hodgkin's disease. Ann. N.Y. Acad. Sci. 73, 335.

Kassel, R. \& Rotrino, A. (1955). Significance of diphtheroids in malignant disease studied by germ-free techniques. A.M.A. Arch. int. Med. 96, 804.

KLieneberger-Nobel, E. (1949). Origin, development and significance of L-forms in bacterial cultures. J. gen. Microbiol. 3, 434. 
KLIENEBERger-Nobex, E. (1951). Filterable forms of bacteria. Bact. Rev. 15, 77.

McDermott, W. (1958). Microbial persistence. Yale J. Biol. Med. 30, 257.

NeIson, J. B. (1936). Studies on an uncomplicated coryza of the domestic fowl. VII. Cultivation of the coccobacilliform bodies in fertile eggs and in tissue cultures. J. exp. Med. 64, 749.

Pappenhemer, A. M., Mollox, E. \& Rose, H. M. (1945). Presence of granules resembling elementary bodies in yolk cells of normal eggs. Proc. Soc. exp. Biol., N.Y. 58, 313.

Spiegelman, S., Aronson, A. I. \& FrTz-J Ames, P. C. (1958). Isolation and characterization of nuclear bodies from protoplasts of Bacillus megaterium. J. Bact. 75, 102.

Van Herick, W. \& Eaton, M. D. (1945). An unidentified pleuropneumonia-like organism isolated during passages in chick embryos. J. Bact. 50, 47.

Warren, J., WitTler, R. G. \& Vincent, M. (1955). Cultivation of tissues in small sealable dishes. J. Lab. clin. Med. 46, 144.

WrTtLer, R. G. (1952). The L-form of Haemophilus pertussis in the mouse. J. gen. Microbiol. 6, 311.

WitTler, R. G., Cary, S. G. \& Lindberg, R. B. (1956). Reversion of a pleuropneumonialike organism to a corynebacterium during tissue culture passage. J.gen. Microbiol. 14, 763.

\section{EXPLANATION OF PLATES}

\section{Plate 1}

Fig. 1. Tissue culture of chick CAM cells showing clusters of inclusions along the periphery of the cell cytoplasm. These contaminants were present in the uninoculated eggs of various flocks from the Washington-Maryland area. Giemsa stain; $\times 1116$.

Fig. 2. Patient's blood cultured in a bottle of TSB. The small white fluffy balls of growth appeared after several days of incubation. $\times 1 \cdot 1$.

Fig. 3. Gram-positive bacillary form of the corynebacterium isolated from the patient's blood in TSB. Gram stain; $\times 1333$.

Fig. 4. Filamentous and pleomorphic Gram-negative forms of the corynebacterium isolated from the patient's blood after transfer to blood agar plates. Gram strain; $\times 1333$.

\section{Plate 2}

Fig. 5. Surface of agar plate inoculated with patient's blood showing the debris of disintegrated WBC after 5 days of incubation. The arrow indicates the area below which the deep colony of granules developed. Dienes's stain and phase contrast; $\times 1013$.

Fig. 6. Same as Fig. 5 but with the focus adjusted slightly below the surface of the agar. The arrow indicates the upper level of the deep colony of granules. Dienes's stain and phase contrast; $\times 1013$.

Fig. 7. Same as Fig. 5 but with the focus adjusted 8-10 $\mu$ below the surface of the agar. The arrow indicates the deep colony of granules at the level of most dense growth. Dienes's stain and phase contrast; $\times 1013$.

Fig. 8. 'Fried egg' type of colony showing large bodies, vacuoles and granules which made up the peripheral portion of the colony. The granular central portion of the colony remained on the agar when the impression preparation was made, thus leaving an empty hole in the centre of this specimen. Giemsa stain; $\times 1080$.

Fig. 9. Small round inclusion bodies (indicated by arrows) in the cytoplasm of WBC of the patient's blood. In the upper left corner of the photograph is an eosinophil containing the denser and highly refractile normal cytoplasmic granulation for comparison. Dienes's stain and phase contrast; $\times 1013$.

Fig. 10. CAM tissue culture inoculated with the patient's blood (4th serial CAM passage). Clusters of inclusions are found along the periphery of the cell cytoplasm. Giemsa stain; $\times 1230$.

\section{Prate 3}

Fig. 11. A chain of three round bodies. Two are still connected by thin protoplasmic material. Several bodies show differentiation of internal contents. Four-day-old culture in VIB + Th(BBL) + AscFl + penicillinase. Hanging drop, unstained, phase contrast; $\times 1029$. 
Journal of General Microbiology, Vol. 23, No. 2

Plate 1

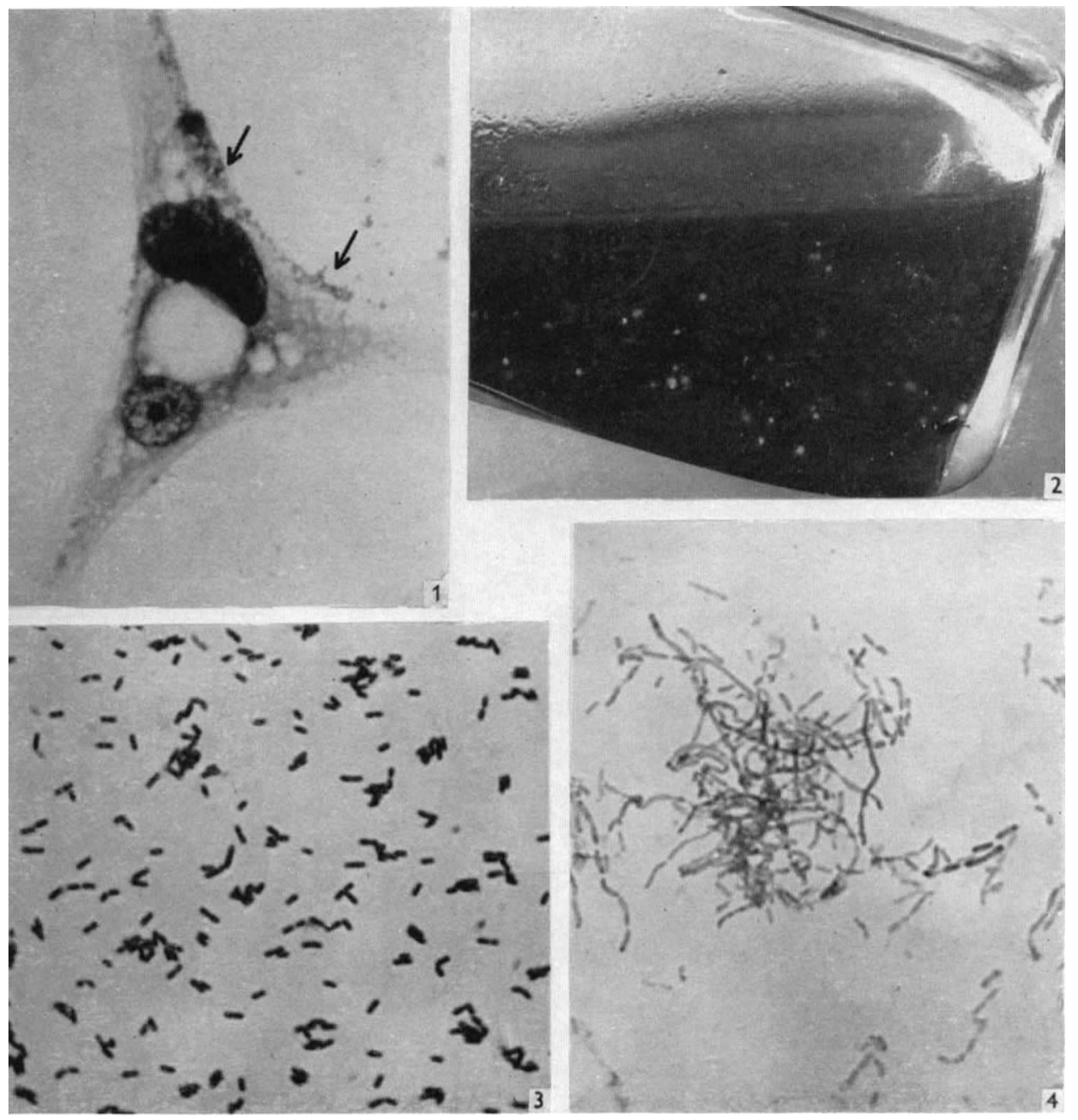



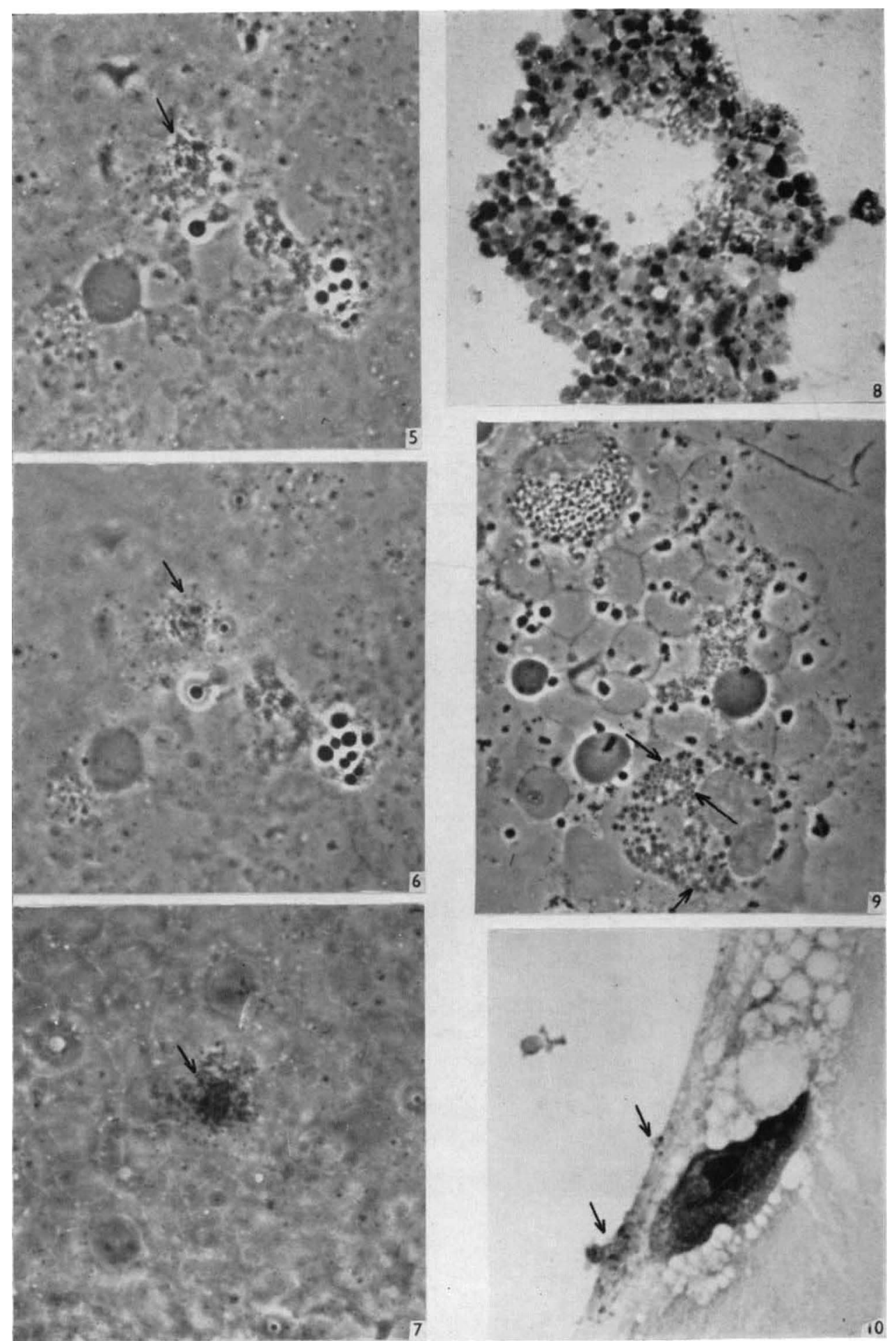
Journal of General Microbiology, Vol. 23, No. 2

Plate 3
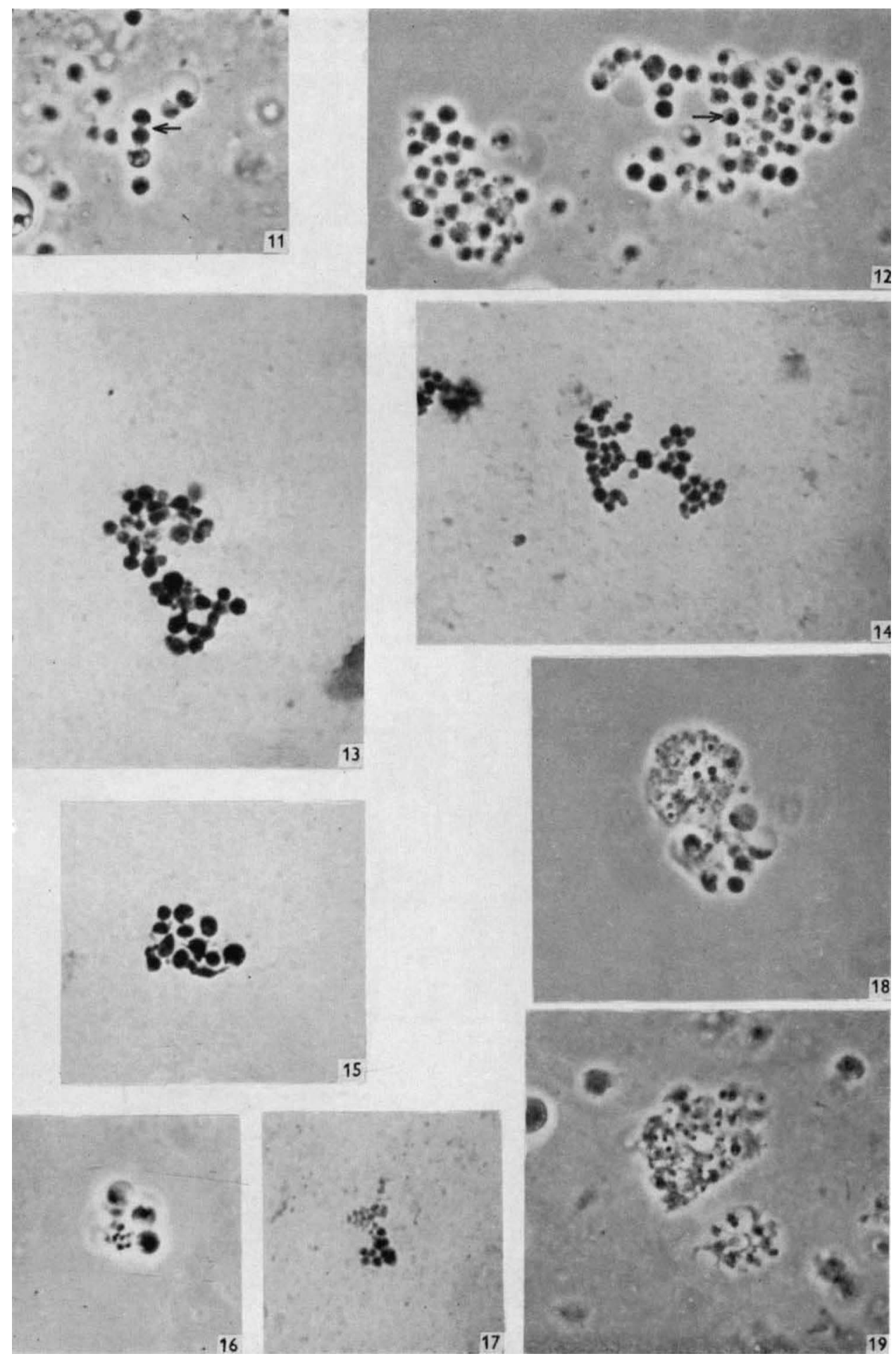

R. G. WITTLER AND OTHERS 

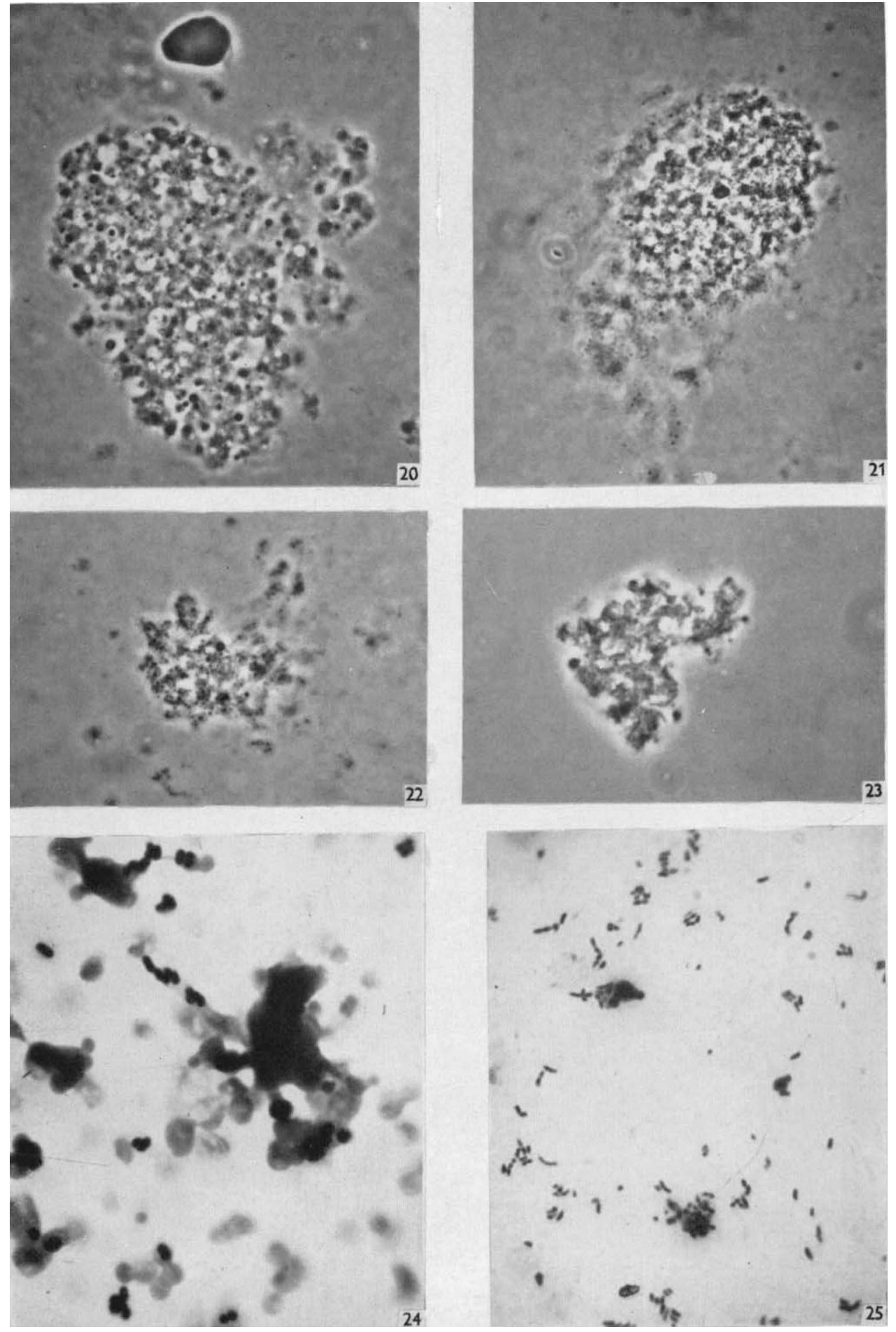

R. G. WITTLER AND OTHERS 
Fig. 12. Large clusters of round bodies. Some show dark caps on a clear bubble; others show differentiation of internal contents; still others are breaking down and merging into delicate masses of amorphous material. Four-day-old culture in $\mathrm{VIB}+\mathrm{Th}(\mathrm{BBL})+\mathrm{Asc} \mathrm{Fl}+$ penicillinase. Hanging drop, unstained, phase contrast; $\times 1029$.

Fig. 13. Cluster of round bodies becoming irregular in shape. Several show the early stage of formation of a protrusion or small bud. Four-day growth in VIB + Th(BBL) + Asc Fl + penicillinase. Giemsa stain; $\times 1080$.

Figs. 14, 15. Clusters of bodies showing fine thread-like forms extending from the bodies. Thirty-six hr. growth in PPLOFl+AscFl. Giemsa stain; $\times 1080$.

Fig. 16. A group of three round bodies and a cluster of granules of various sizes. Sixty hr. culture in PPLOFl + Asc Fl + penicillinase. Hanging drop, unstained, phase contrast; $\times 1346$.

Fig. 17. A cluster of delicate tiny granule-like round and oval forms lying next to a group of round bodies. Eight day growth in PPLOFl + AscFl. Giemsa stain; $\times 1080$.

Fig. 18. A group of round bodies in the process of breaking down and merging, and the development of an amorphous mass filled with granules of various sizes. Sixty hr. culture in PPLOFl+ Asc Fl + penicillinase. Hanging drop, unstained, phase contrast; $\times 1187$.

Fig. 19. Amorphous mass filled with granules. Traces of the bubbles from the round bodies are still distinguishable. Four-day-old culture in Th(BBL) + Rabbit S. Hanging drop, unstained, phase contrast; $\times 1029$.

\section{Puate 4}

Fig. 20. Development of granules within a large amorphous mass composed of disrupted bodies, structureless protoplasmic material, and bubbles. Four-day-old culture in Th(BBL)+Rabbit S. Hanging drop, unstained, phase contrast; $\times 1029$.

Fig. 21. An advanced stage of development of granules within an amorphous protoplasmic mass. The second passage of the patient's blood in PPLOFl + AscFl was plated on VIA + AscFl and the 3-day-old growth on this plate photographed. Agar block, Dienes's stain, phase contrast; $\times 1029$.

Fig. 22. A stage shortly before reversion to bacilli. The granules become arranged in orderly fashion and appear as beads on an extremely fine thread. Culture same as Fig. 18. Agar block, Dienes's stain, phase contrast; $\times \mathbf{1 5 8 3}$.

Fig. 23. The final stage before reversion to bacilli. The granules are relatively less prominent, and very delicate, filamentous bacillary forms are discernible throughout the mass. Twenty-four hr. growth of the second passage of the patient's blood in PPLOFl+ Asc Fl. Hanging drop, unstained, phase contrast; $\times 1583$.

Fig. 24. Corynebacterium sp. (strain 6-4a) immediately after its reversion to bacillary form. Coccoid and diplococcal forms are common at this time. Twenty-four hr. growth of the fourth passage of the patient's blood in VIB + AscFl. Gram strain; $\times 1506$.

Fig. 25. Reversion of Corynebacterium sp. (Strain 11-4d) from transitional form. Eleven-day growth in $T h(B B L)+$ Rabbit $S$ inoculated with the patient's blood. Giemsa stain; $\times 1080$. 